\section{Compendium of Polymer Terminology and Nomenclature}

(IUPAC Recommendations 2008) "The Purple Book", $2^{\text {nd }}$ Ed., Richard G. Jones, Edward S. Wilks, W. Val Metanomski, Jaroslav Kahovec, Michael Hess, Robert Stepto, Tatsuki Kitayama (Editors) RSC Publ. 2009, 464 p

ISBN: 978-0-85404-491-7

The second edition of the Purple Book is an extension and revision of the earlier compendium published in 1991 by the Polymer Division (IV) of the International Union of Pure and Applied Chemistry (IUPAC). The present compendium contains 22 chapters divided into two sections: Terminology (13 chapters) and Nomenclature ( 9 chapters). In comparison with the first edition, which contained 9 chapters, the scope of this new one has been more than doubled. Only four of the chapters of the early edition have been retained in their original form (Chapters: 2, 3, 6 and 19) and one chapter has been excluded.

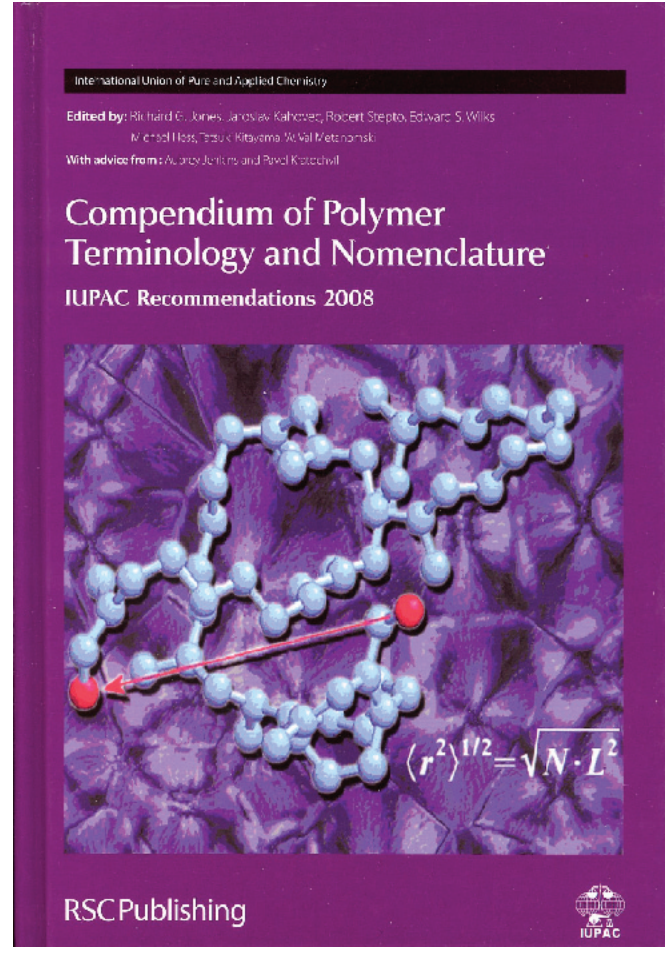

The Content of The Purple Book, $2^{\text {nd }}$ Edition, 2009

IUPAC Polymer Division (IV). History. Membership

\section{Terminology}

1. Glossary of Basic Terms in Polymer Science (1996)

2. Stereochemical Definitions and Notations Relating to Polymers (1980)

3. Definition of Terms Relating to Individual Macromolecules, their Assemblies, and Diluted Polymer Solutions (1988)

4. Basic Classification and Definitions of Polymerization Reactions (1994)

5. Definitions Relating to Stereochemically Asymmetric Polymerizations (2002)

6. Definitions of Terms Relating to Crystalline Polymers (1988)

7. Definitions of Terms Relating to Low-Molar-Mass and Polymer Liquid Crystals (2001)

8. Definitions of Terms Relating to the Non-Ultimate Mechanical Properties of Polymers (1998)

9. Definition of Terms Related to Polymer Blends, Composites, and Multiphase Polymeric Materials (2004)

10. Terminology of Polymers Containing Ionisable or Ionic Groups and of Polymers Containing Ions (2006)

11. Definition of Terms Relating to the Structure and Processing of Sols, Gels, Networks and Inorganic-Organic Hybrid Materials (2007)

12. Definition of Terms Relating to Reaction of Polymers and to Functional Polymeric Materials (2003)
13. Definition of Terms Relating to Degradation, Aging, and Related Chemical Transformations of Polymers (1996)

\section{Nomenclature}

14. Introduction to Polymer Nomenclature

15. Nomenclature of Regular Single-Strand Organic Polymers (2002)

16. Nomenclature of Regular Double-Strand (Ladder and Spiro) Organic Polymers (1993)

17. Structure-Based Nomenclature for Irregular Single-Strand Organic Polymers (1994)

18. Graphic Representations (Chemical Formulae) of Macromolecules (1994)

19. Source-Based Nomenclature for Copolymers (1985)

20. Source-Based Nomenclature for Non-Linear Macromolecules and Macromolecular Assemblies (1997)

21. Generic Source-Based Nomenclature for Polymers (2001)

22. Abbreviations

\section{Appendix}

Bibliography of Biopolymer-Related IUPAC-IUBMB Nomenclature Recommendations

Index 


\section{History and Membership}

IUPAC is a scientific, international, non-governmental and objective body addressing global issues involving different areas in chemical science. Polymer Division (Division IV) is one of eight IUPAC's divisions and the only worldwide organization concerned with defining and leading developments in polymer science and technology. Its activity began sixty years ago when several visionary leaders in polymer chemistry such as J. J. Hermans, M. L. Higgins, O. Kratky, H. F. Mark, G. Natta recognized the need for polymer language, and as members of the Subcommission on Nomenclature of the IUPAC Commission on Macromolecules, published (in 1952) the first system for naming macromolecular compounds and the use of symbols and terms in polymer science. Although the system was only partially accepted, it drew attention in the field and paved the way to the development of modern polymer nomenclature and terminology.

Rapid advancement in polymer science and technology has brought about changes in titles of the Division's bodies increasing the number of projects and collaborators.

The chronology of titles: Commission on Macromolecules with Subcommission on Nomenclature in 1952; the Macromolecular Division (IV) with Commission on Macromolecular Nomenclature in 1968; the Macromolecular Division (IV) with Subcommittee on Macromolecular Terminology (IV.1) in 2002; Polymer Division in 2004 and Subcommittee on Polymer Terminology in 2005.

The current Polymer Division (IV) runs more than 100 projects in six main areas-subcommittees: Polymer Terminology; Developing Polymer Materials; Polymer Education; Molecular Characterization of Polymers; Structure and Properties of Commercial Polymers; Modeling of Polymerization Kinetics. Since its beginning, Division IV has attracted many scientists from academia, the publishing firms, and the polymer industry all over the world. Thanks to the devoted work of many members and under skilful chairmanship, the Division IV has come to be one of the most active IUPAC divisions.

The Subcommittee on Polymer Terminology is responsible for defining terminology and standards in macromolecular and polymer science and technology. It continues to work on macromolecular nomenclature in collaboration with the new Division VIII (Division of Chemical Nomenclature and Structure Representation founded in 2002 to deal with nomenclature in an integrated manner across all branches of chemistry). More than one third of the Division's projects are the activity of this Subcommittee including much interdivisional collaborations. The results are a series of documents published as recommendations in IUPAC's official journal Pure and Applied Chemistry. The new edition of the Purple Book collects in a single volume the most important IUPAC recommendations of polymer terminology and nomenclature. Many new documents as well as the revisions of those already published are in various stages of preparation.

\section{Terminology}

Modern polymer science and technology has an interdisciplinary nature. Polymer science is a blend of organic chemistry, physical chemistry, materials chemistry, materials physics, inorganic chemistry, statistics... while polymer technology is a combination of polymer science with chemical engineering, mechanical engineering, and rheology. With broadening the research areas, the polymer science and technology become so diverse and interwoven with other modern scientific and technological disciplines demanding for unique and easily understood terms. IUPAC's answers are in the new "Subcommittee on Polymer Terminology", increased number of terminology documents and frequent collaborations among various very specific fields.

Chapter 1 is the most fundamental document as it contains definitions of 135 basic terms in polymer science. The terms are divided in three sections: molecules and molecular structures (e.g. macromolecule or polymer molecule, constitutional unit, tacticity, chain, branch, star macromolecule, network, etc), substances (e.g. monomer, oligomer, polymer, copolymer, branched polymer, star polymer, polymer blend, etc.), and reactions (e.g. polymerization, copolymerization, polycondenzation, polyaddition, ionic polymerization, depolymerization, etc.). This document is the third revision of the earlier IUPAC recommendations published in 1952 and 1974 years.

The discovery of stereoregular polymerizations (Ziegler, Natta, 1952-1955) and the possibility of controlling a chain structure and tacticity increased the interest in polymer stereochemistry resulted in the first stereochemical definitions and notations relating to polymers (1962). Chapter 2 is a complete revision of the first document. It provides detailed definitions and illustrations of the most important features corresponding to stereoregularity in polymers such as: constitutional and configurational units, tacticity, sequences and their relative configurations, conformations of polymer molecules and specific terminology for crystalline polymers.

Chapter 3 deals with terminology in key areas of the physical chemistry of macromolecules. More than 122 definitions and symbols relate to individual macromolecules (e.g. molar mass, M; degree of polymerization, DP or X; random coil; end-to-end distance, r;....), their assemblies (e.g. heterogeneity, uniform polymer, molar-mass average, $\mathrm{M}_{\mathrm{k}}$, average degree of polymerization, $\mathrm{X}_{\mathrm{k}}$; distribution function, ...), and diluted polymer solutions including thermodynamic terms, transport properties, terms relating to scattering and separation. Better understanding of physical properties of macromolecules in concentrated solutions and in bulk, and improper use of some terms are the reasons why revision and extension of this document are in progress.

The basic definitions of terms relating to polymerization reactions are presented in Chapters 4 and 5. Chapter 4 describes the clear differences among chain polymerization, condensative chain polymerization, polycondenzation and polyaddition. Chapter 5 defines terms relating to polymerization processes that involve chirality and proceeds in an unsymmetrical manner under of chiral features present in monomer, initiator, and catalyst or solvent.

Definitions and some illustrations for 54 terms relating to the structure of crystalline polymers, crystallization kinetics, local conformations and morphology within polymer crystals are described in Chapter 6. The revision of this document is in progress taking into account the development and results of new experimental techniques.

Terminology for the specific area of low molar mass and polymer liquid crystals, Chapter 7, was prepared by joint efforts of working group of IUPAC's Commission on Macromolecular Nomenclature and members of the International Liquid Crystal Society. As both classes of liquid crystals have common terminology, the document offers definitions for 128 selected terms for types of mesophases, structure, texture, defects and physical characteristics of liquid crystals and polymer liquid crystals accompanied with a number of illustrations, recommended abbreviations and symbols.

The first collection of 72 terms met in the conventional mechanical characterization of polymeric materials is the subject of Chapter 8. Names, definitions, mathematical expressions, symbols and units for phenomena (stresses, strains, de- 
formations...) in elastic and viscoelastic behavior of liquid or solid polymeric materials prior to failure are recommended.

Terms most commonly used in the field of multiphase polymeric systems, blends and composites are described in Chapter 9. Nearby basic terms such as miscibility, compatibility, dispersion, additive, filler, laminate, etc. the document defines terms related to phase domain behavior and domain morphology, all together 90 terms have been defined.

Differences among various types of ion-containing polymers regarding the polymer structure and the nature, content and location of ionic groups are defined in Chapter 10 .

Chapter 11 was prepared as a joint project of Inorganic (II) and Polymer Divisions (IV). It is a glossary of terms relating to the structure and processing of inorganic, polymeric and inorganic-organic hybrid materials from starting materials through gels to solid products. Definitions of 196 terms of this document are divided in four sections: precursors, gels, solids, and processes.

Specific polymer reactions are treated in Chapters 12 and 13. Growing production and application of functional polymers claim for clear terminology in the field. Definitions of 64 general and specific terms (Chapter 12) are divided in three sections: reaction involving polymers; polymer reactants and reactive polymeric materials; functional polymeric materials. Predominantly spontaneous changes in structure and properties of polymeric materials owing to physical or chemical factors such as heat, light, load, radiation, microorganisms are important from the economic and environmental point of view. Definitions of 39 basic terms like aging, degradation, crazing, stability, carbonization, etc. are the subject of Chapter 13 .

\section{Nomenclature}

The main purpose of standardized or universally understood nomenclature is to improve communication among the scientific community and to connect the known structure of a polymer with its name. The need for a systematic nomenclature (i.e. nomenclature based on convention with a number of principles and rules) is even more important considering the rapid development of polymers from predominantly linear and randomly branched polymers at the beginning (1950) to a bewildering variety of nonlinear polymers like comb, star, hyperbranched, denritic, catenanes, rotaxanes, knots, and their combinations.

Nine chapters in the new "Purple Book" deal with three methods of polymer nomenclature: structure-based, source-based, and abbreviations. While the structure-based and source-based nomenclature are elaborated in details, the list of abbreviations for names of polymer substances (Chapter 22) is taken over from the International Organization of Standards (ISO) and its nomenclature is not in accord with IUPAC recommendations.

"Nomenclature of regular single-strand organic polymers", Chapter 15, represents the third improved edition of the document (IUPAC recommendation 1975, ACS recommendations from 1968) containing fundamental principles and rules for structure-based nomenclature of polymers. The polymer is named as "poly(constitutional repeating unit)" or "poly(CRU)". The preferred CRU is named as a bivalent organic group according to the recent nomenclature rules for organic chemistry. In order to draw and name the correct CRU, a number of rules should be applied following three steps: identification, orientation, and naming. The rules are mainly related to the order of seniority of individual parts of the CRU (atoms, subunits, substituents, and bonds). The structure-based naming is illustrated on 79 examples of polymer names. The document also contains a long list of names of common subunits, as well as structureand source-based names for common polymers.
The principles of structure-based nomenclature have been extended to regular double-strand (ladder and spiro) polymers, irregular single-strand organic polymers, and copolymers having regular structure.

In double-strand polymer, Chapter 16, the macromolecules consist of an uninterrupted sequence of rings with adjacent rings having one atom in common (a spiro polymer) or two or more atoms in common (a ladder polymer). Since the polymer has a sequence of rings, a preferred CRU is identified (oriented and named) by conceptual breaking of the ring's bonds following specific rules for complex rings systems. Depending on the complexity of the double-strand polymer, the CRU can be a tetravalent group or the unit may be hexavalent or octavalent. End groups, when known, are specified by appropriate substituent prefix names, together with Greek letters $(\alpha-, \alpha$ and $\left.\omega-, \omega^{\prime}-\right)$ in front of the name of the polymer. For example:

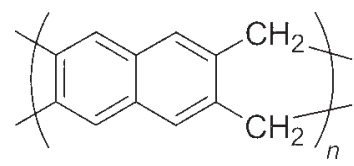

poly(naphtalene-2,3:6,7-tetrayl-6,7-dimethylene)

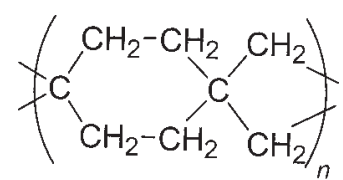

poly(cyclohexane-1,1:4,4-tetrayl-4,4-dimethylene)

Rules for structure-based naming of irregular single-strand polymers are described in Chapter 17. Irregular single-strand polymers (also oligomers and blocks) consist of more than one type of CRU. These are, for example, partially modified polymers, polymers from a single monomer, which can polymerize in different ways, block and graft copolymers, or homopolymers having both head-to-tail and head-to-head arrangement of monomeric units. They are named by placing the prefix "poly" (or "oligo") before the structure-based names of irregularly repeating constitutional units. The names of individual CRUs are separated by oblique strokes and enclosed in parentheses. In block copolymers, the blocks are bonded by using long dashes, while in graft copolymers the grafted chains are treated as substituent to the main chain.

Chemical formulae or graphic representations of chemical compounds are the international language of chemistry. In comparison with chemical formulae of low molecular weight compounds, chemical formulae of polymers must additionally reflect the multiplicity of CRUs in the macromolecule and various possibilities for connecting the CRUs in a macromolecule. The document in Chapter 18 provides rules and examples for the graphic representation of CRUs, monomeric units, regular and irregular polymers and various copolymers (alternating, periodic, statistical, random, block, graft, etc.). Particularly emphasized is that the graphic representation (formula) of CRU should be in accordance with the naming rules of CRU (identification, orientation, naming).

The source-based nomenclature was applied to homopolymers, copolymers (Chapter 19) and to nonlinear macromolecules and macromolecular assemblies (Chapter 20). The principle for writing the source-based name of a polymer is to put the prefix "poly" in front of the name of the monomer, e.g. polystyrene. When the name of the monomer consists of more than one word, its name is parenthesized, e. g. poly(vinyl chloride). 
The main shortcomings of source-based nomenclature are structural differences between the monomer unit in the polymer (e.g. $-\mathrm{CHX}-\mathrm{CH}_{2}$ ) and in the monomer (e. g. $\mathrm{CHX}=\mathrm{CH}_{2}$ ) as well as the same name for polymers having different structure and properties but obtained from the same monomer in different polymerization conditions. For example, polybutadiene is the name for polymers 1,2-, 3,4-, cis-1,4-, trans-1,4- polybutadiene. The name poly(vinyloxirane) does not indicate whether the vinyl group (1) or the cyclic ether group (2) was the focus of polymerization. The utility of generic source-based nomenclature (Chapter 21), i.e. the addition of a polymer class name to the source-based name of the monomer, can contribute to clear the polymer structure:

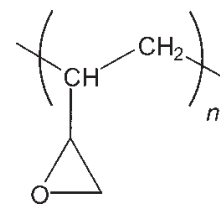

(1) polyalkylene:vinyloxirane<smiles>C=CC(CC(C)(C)C)OC(C)(C)C</smiles>

(2) polyether:vinyloxirane

In naming copolymers, the source-based names of various monomer units are connected using seven different infixes, written in italic, which define their way of arrangements: $-\mathrm{Co}$-, -stat-, -ran-, -alt-, -per-, -block-, -graft-.
For example

- poly[styrene-co-(methyl metacrylate)]

- polybutadiene-graft-polystyrene

Similarly, nonlinear macromolecules (cyclic, branched, blocked, grafted) and macromolecular assemblies (crosslinked, interpenetrated, complexed) are classified and named by addition of corresponding italicized qualifiers such as: cyclo, branch, comb, star, net, ipn, sipn, and compl. For example:

- (net-polystyrene)-blend-(net-polybutadiene) blend of two networks

- poly(acrylic acid)-compl-poly(4-vinylpyridine) complex of two homopolymers

Simplicity, direct relation to monomer names and the historical trivial names are the reasons that the source-based nomenclature is still firmly entrenched in the scientific literature and in praxis. Therefore, after long delaying, the Commission decided to recommend source-based nomenclature (2001) as an alternative official nomenclature.

The global increase in the production and consumption of polymers and the presence of polymer science and technology at the center of numerous new developments lead to conclusion that the $21^{\text {st }}$ century is the Polymer Age. Although practice precedes theory, IUPAC has made enormous progress through the years in providing systematic nomenclature and terminology. The new edition of the Purple Book gathers in a single volume key IUPAC nomenclature and terminology documents that will contribute to clear and precise communications for those working in this field.

Nearly all IUPAC documents are translated into the Croatian language and published in the journal "Kemija $u$ industriji". Only translated Chapters 11,14 and 22 are waiting to be published.

Dr. sc. Vida Jarm 